\title{
One Dimensional Simulations of Transients in Heavy Ion Injectors*
}

\author{
J.J. Barnard, G.J. Caporaso \\ Lawrence Livermore National Laboratory, L-440, Livermore, CA 94550
}

\author{
S.S. Yu, and S. Eylon \\ Lawrence Berkeley Labooratory, MS 47-112, Berkeley CA 94720
}

\begin{abstract}
A fast-running time-dependent one-dimensional particle code has been developed to simulate transients in both electrostatic quadrupole and electrostatic column heavyion injectors. Two-dimensional effects are incorporated through the use of an approximation to the transverse part of the Laplacian operator. Longitudinal electric fields are solved on a mesh. An external circuit is coupled to the column, and the effect of the beam on the circuit is modeled. Transients such as initial current spikes, space-charge debunching, and beam loading of the circuit, are simulated. Future directions for the code include introduction of envelope and centroid equations to provide beam radius and displacement information and the modeling of secondary electron currents arising from beam-spill.
\end{abstract}

\section{INTRODUCTION}

We have used the 1-dimensional, time-dependent code HINJ (for Heavy-ion INJector) in order to study transient effects in both the electrostatic quadrupole (ESQ) and electrostatic column (ESAC) heavy ion injector configurations. The ESAC uses an increasing longitudinal accelerating electric field which causes a radial convergence of the electric field lines, and hence a focusing component to the electric field. The ESQ uses alternating gradient electrostatic quadrupoles to provide the focusing. The most accurate simulations have been done using 2-D codes (e.g GYMNOS, [1]) in the case of the axisymmetric ESAC or the axisymmetric diode in an ESQ, or 3-D codes (e.g WARP3d, [2]) for the non-axisymmetric ESQ. However, it has been valuable for physical understanding, to develop a fast running $1 \mathrm{D}$ code which examines longitudinal dynamics, and thus shed light on the transient behavior of the injector.

In this paper, we first briefly discuss the types of transients that may be investigated by using the code HINJ. The physics which is included in HINJ is discussed in section III. In section IV we provide an example of a benchmark of the code against a diode experiment, and an example of code results for one possible ESQ design that is being planned for the Induction Linac Systems Experiment (ILSE) [3] now being planned for the Lawrence Berkeley Laboratory (LBL).

${ }^{*}$ Work performed under the auspices of the U.S.

D.O.E. by LLNL under contract W-7405-ENG-48 and by

LBL under contract DE-AC03-76SF00098.

\section{TYPES OF TRANSIENTS}

We divide injector transients that may be modeled using HINJ into at least four classes: 1 . initial current spikes, 2. space-charge debunching, 3 . beam loading of the circuit, and 4. secondary electron currents arising from beam spilling. Initial current spikes arise because during the initial transit of the beam across the injector, the space charge electric fields are not the same as those that occur during the "D.C." part of the pulse. In particular, during the initial part of the pulse there is no space charge in front of the head of the beam so that the electric fields are larger than the steady state fields, and so more current is drawn from the emitting surface than occurs in steady state. This oversupply of space-charge inhibits emission from the emitter and produces a paucity of current following the current spike, which then induces an over-supply and so forth. These oscillations are damped on approximately the transit time scale after which steady state conditions are reached. Space-charge debunching is a result of the line-charge density gradients at the head and tail of the beam. These gradients result in an electric field which causes the head and tail of the pulse to expand relative to the center, and causes the length of the flat top in the current pulse to decrease in duration as rarefaction waves propagate inward from the head and tail. Beam loading arises as the magnetic field of the beam induces return currents, and the electric field of the beam induces image charges, in the accelerating structure. These act as current and voltage sources in the circuit, altering the voltages on the accelerating electrodes, which in turn alters the beam current. Finally, if errant beam particles strike an electrode, electrons may be emitted and accelerated producing large current perturbations, which will again alter the accelerating electrode voltages.

\section{THE PHYSICS OF HINJ}

As stated earlier, HINJ is a 1-D (z), electrostatic, time-dependent particle code. The particles are moved using the non-relativistic equations of motion:

$$
\frac{\mathrm{d} z_{i}}{\mathrm{~d} t}=v_{i} \quad \text { and } \quad \frac{\mathrm{d} v_{i}}{\mathrm{~d} t}=-\frac{q e}{m} \frac{\partial \phi}{\partial z}
$$

where $q$ is the charge state of the ions, $e$ it the proton charge, $z_{i}$ and $v_{i}$ are the longitudinal position and velocity of the $i^{t h}$ ion, and $m$ is the ion mass. The on-axis potential $\phi$ is solved on a grid. In diode geometries, however, there is an option which allows calculation of the potential by summation of the known greens function for each particle. In the multi-electrode geometries used in the ESAC and 
ESQ the calculational grid is used. One novel feature of the code, is the incorporation of 2-dimensional effects by the use of an approximation to the transverse part of the Laplacian operator [4].:

$$
\nabla^{2} \phi \cong \frac{\partial^{2} \phi}{\partial z^{2}}+\frac{V_{e x t}-\phi}{a_{*}^{2}}=-\frac{\rho}{\epsilon_{0}}
$$

Here, $a_{*}^{2} \equiv(1+g) a^{2} / 4, g \equiv 2 \ln r_{p} / a, \phi$ is the electrostatic potential on axis, $V_{\text {ext }}$ is the external potential at the pipe radius $r_{p}$ and $a$ is the beam radius. The equation reduces to the correct expression in the two extreme limits:

$$
\begin{array}{ll}
\frac{\partial^{2} \phi}{\partial z^{2}} \cong-\frac{\rho}{i_{0}} & \text { if } L_{\nabla}<<a_{*}, \text { and } \\
\phi \cong V_{e x t}+\frac{(1+g) \lambda}{4 \pi \epsilon_{0}} & \text { if } L_{\nabla}>>a_{*}
\end{array}
$$

Here $L_{\nabla} \supseteqq \phi /|\partial \phi / \partial z|$. Since in both the ESAC and ESQ, the beam has much smaller radius than length, the beam rapidly transfers from the $L_{\nabla}<<a_{*}$ regime to the $L_{\nabla}>>a_{*}$ regime and the 2-D equation is essential in order to get meaningful computational results. One may get a qualitative understanding of the two dimensional effects, by examining the greens function for eq. (1) in the diode geometry. A charge slab of unit total charge with radius $a$, at position $z_{p}$ in a diode of length $d$ with external voltage $V_{e x t}=V_{o}(1-z / d)$, has a solution to eq. (2) which satisfies:

$$
\phi_{s c}= \begin{cases}\frac{a_{*} \sinh \left(z / a_{*}\right) \sinh \left(\left[d-z_{p}\right] / a_{*}\right)}{\epsilon_{0} \sinh \left(d / a_{*}\right)} & z<z_{p} \\ \frac{a_{*} \sinh \left(z_{p} / a_{*}\right) \sinh \left([d-z] / a_{*}\right)}{\epsilon_{0} \sinh \left(d / a_{*}\right)} & z>z_{p}\end{cases}
$$

Here $\phi_{s c} \equiv \phi-V_{e x t}$. For the case $a_{*} \longrightarrow \infty$, The 1-D greens function is obtained:

$$
\phi_{s c}= \begin{cases}z\left(d-z_{p}\right) /\left(\epsilon_{0} d\right) & z<z_{p} \\ z_{p}(d-z) /\left(\epsilon_{0} d\right) & z>z_{p}\end{cases}
$$

In the 1-D case, the contribution to the space charge field from a particle falls off linearly with distance from the charge sheet, vanishing at $z=0$ and $z=d$, while the greens function falls off exponentially after a distance from the charge sheet $\sim a_{*}$ in the 2-D case. This indicates that the effect of the radial boundaries, i.e. image charges on the walls, limits the extent over which the space charge has a significant influence. This affects the initial transients, since the relative transit time in the 2-D case is approximately $a_{*} / v$ rather than $d / v$, and $v$ is an average velocity.

The external voltage at various discrete locations in $z$ is determined by a circuit equation. The external circuit is divided into a Marx bank power supply and accelerating column, which accelerates the beam by creating a decreasing voltage at discrete electrodes along the column. The equivalent circuit is shown in figure 1 [5]. The potential and current of the beam act as voltage and current sources in the circuit [6]. For example, the equation for relating the voltages on $i^{t h}-1$ through $i^{\text {th }}+1$ electrode is given by:

$$
C_{p i} \frac{\mathrm{d} V_{i-1}}{\mathrm{~d} t}-\left(C_{p i}+C_{p i+1}+C_{s i}+C_{g i}\right) \frac{\mathrm{d} V_{i}}{\mathrm{~d} t}+C_{p i+1} \frac{\mathrm{d} V_{i+1}}{\mathrm{~d} t}
$$

$=-\frac{V_{i-1}}{R_{i}}+\left(\frac{1}{R_{i}}+\frac{1}{R_{i+1}}\right) V_{i}-\frac{V_{i+1}}{R_{i+1}}-N_{b}\left(I_{b i}-I_{b i+1}\right)-C_{g i} \frac{\mathrm{d} \phi_{i}}{\mathrm{~d} t}$

Here $C_{p i}$ is the capacitance between the $i^{t h}-1$ and $i^{t h}$ electrode, (where the emitting surface is the $0^{\text {th }}$ electrode), $R_{i}$ is the resistance between the $i^{t h}-1$ and $i^{\text {th }}$ electrode, $C_{s i}$ is the stray capacitance between the $i^{t h}$ electrode and ground, $C_{g i}$ is the capacitance between the electrode and the on-axis potential, $V_{i}$ is the voltage on the $i^{t h}$ electrode, $I_{b i}$ is the current in a single beam (out of $N_{b}$ beams) at the $i^{\text {th }}$ electrode, $C_{d}$ is the capacitance of the high voltage dome (which is at the voltage of the emitting surface). The external voltage is assumed to linearly vary between electrodes. Wire grids may replace a given electrode (for which $\phi=V_{e x t}$ ).

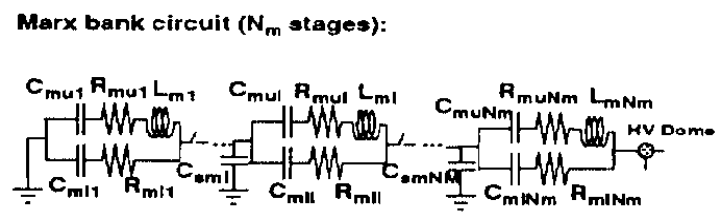

Injector column circuit ( $N$ electrodes):

Beam acts as both current source I,

and voltage source $\mathrm{V}$.

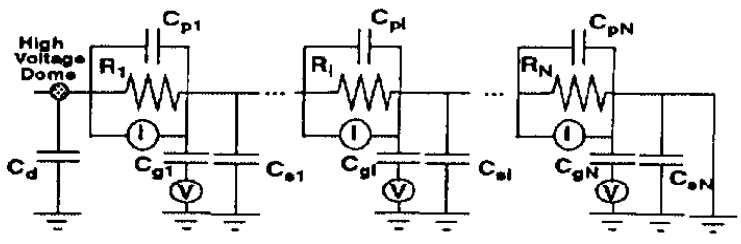

Figure 1. Circuit diagram for marx bank and injector modeled in HINJ.

Other features that are in the process of being incorporated into HINJ, include the following: 1. Envelope equations to self-consistently determine $a_{*} .2$. The effects of spurrious electron currents on the circuit equations, by injection of negative return currents at various electrodes, proportional to the current intercepted at an electrode, as determined by the radius of the electrode and the envelope radius.

\section{EXAMPLES OF RESULTS FROM HINJ}

One test of HINJ was made by comparing the results from a simulation to an experiment performed on the Multi-Beam Experiment at LBL (MBE-4) by one of the authors (S. Eylon). It consisted of a $13.3 \mathrm{~cm}$ diode followed by a $1.8 \mathrm{~m}$ drift section. An aperture at the diode exit reduced the current by a factor of approximately 0.4 . In simulating the experiment, we used a constant beam radius $a$ such that the total current matched the total current in the experiment. Since the diode region did not have a definite outer "pipe" radius, and since $r_{p}$ is defined as that radius at which the external voltage linearly decreases from $V_{0}$ to 0 , we estimate that the equivalent $r_{p} \sim 2 d$ where $d$ is the diode length. Because $r_{p}$ enters in the longitudinal dynamics only logarithmically, the results are not sensitive to the exact value of $r_{p}$. Fig. 2 shows simulation results of 
the current and energy at the diagnostic station (LPO) at the end of the drift region, for two different voltage waveforms applied to the diode, and used as input to HINJ. Figure 3 shows the same quantities as measured in the experiment. The qualitative features of the experiment are seen in the simulation results. (Note that the time axes are displaced by $\sim 2 \mu \mathrm{s}$, reflecting different zero points.)
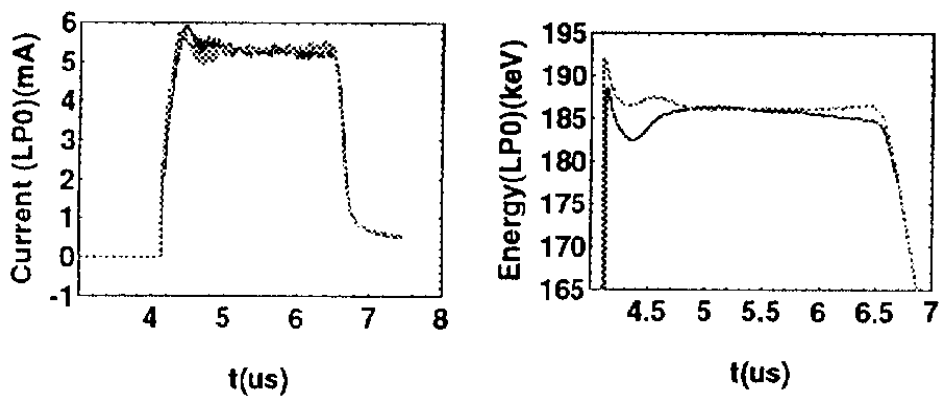

Figure 2. HINJ simulation of an MBE-4 experiment.
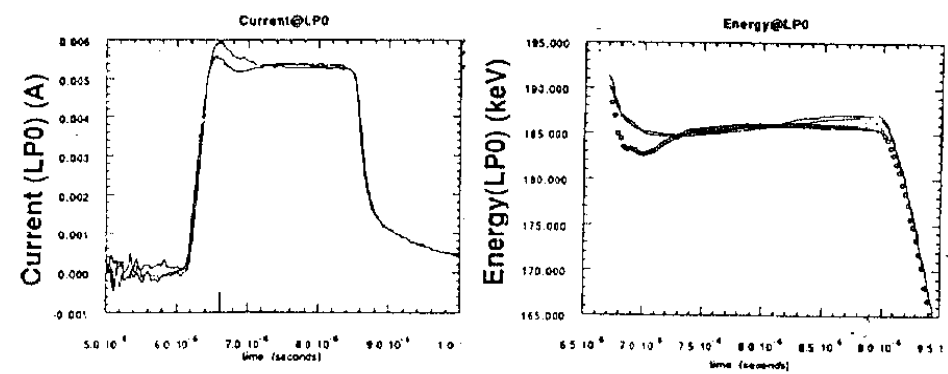

Figure 3. Experimental results of an $\mathrm{MBE}-4$ experiment.

Another example of HINJ results is shown in figs. 4 and 5. A simulation of an ESQ that has circuit parameters which are typical of a possible ILSE [3] injector is shown. Fig. 4 shows the current at the emitter, diode exit, and injector exit. Both the initial current spike, and the erosion of the beam flattop due to space-charge debunching are evident. In fig. 5 the difference in final energy for a beam with and without beam loading of the circuit is shown. Beam loading effects of order ten $\mathrm{keV}$ are apparent. The ESQ column modeled consisted of 11 electrodes and the circuit values used were (cf. fig. 1): $R_{1}=1080 \Omega, R_{2}=$ $216 \Omega, R_{i>2}=432 \Omega, C_{d}=200 \mathrm{pF}, C_{p i}=400 \mathrm{pF}, C_{g i}=$ $10 \mathrm{pF}, C_{s i}=20 \mathrm{pF}$. The Marx bank was modeled as a single stage $\left(N_{m}=1\right)$ with the values: $R_{m u 1}=864 \Omega$, $R_{m l 1}=2070 \Omega, C_{m u 1}=1306 \mathrm{pF}, L_{m 1}=0.0378 \mathrm{mH}$.

\section{CONCLUSIONS}

We have developed a 1-D, time-dependent particle code HINJ in which 2-D effects are incorporated into the code approximately, and in which beam loading is included self-consistently. The code has been compared to analytic computational, and experimental results, and has been found to capture many of the physical details in the transient behavior.

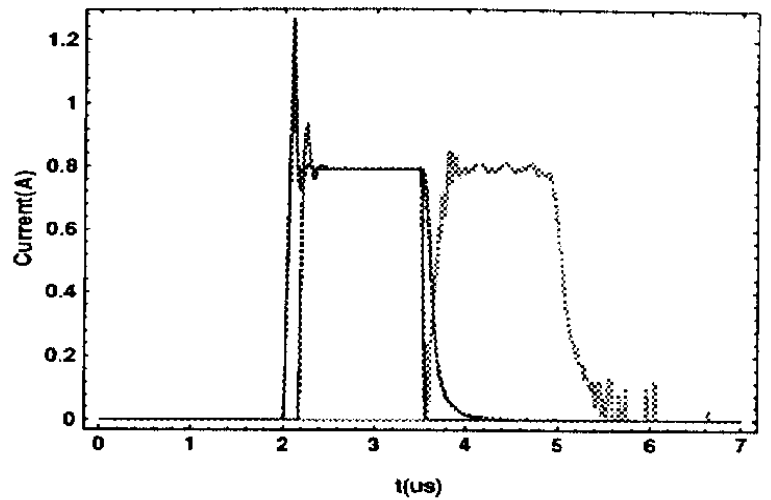

Figure 4. HINJ simulation of ESQ current pulse.

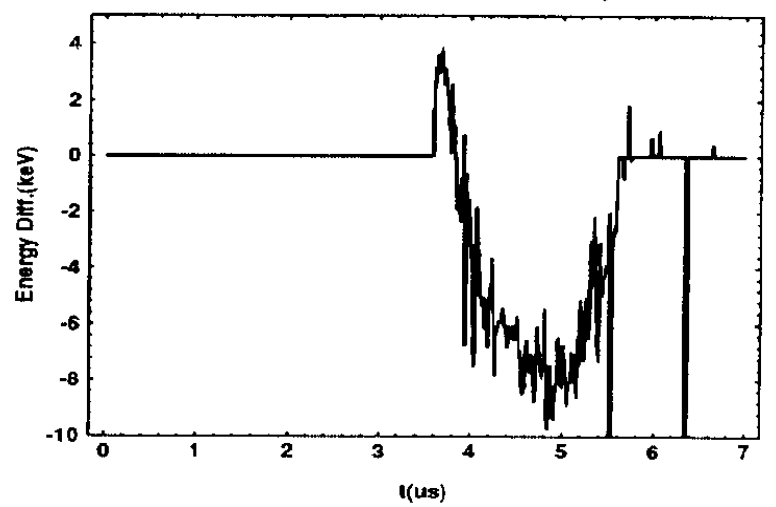

Figure 5. HINJ simulation of the difference in ion final energy between a beam-loaded and unloaded circuit in an ESQ.

\section{ACKNOWLEDGEMENTS}

The authors would like to acknowledge Yu-Jiuan Chen, Andy Faltens, Dennis Hewett, Bruce Langdon, Lou Reginato, and Henry Rutkowski, for the circuits, equations, experimental results, discussions and suggestions which they provided and which contributed substantially to this work.

\section{REFERENCES}

[1] Y.J. Chen, D. Hewett, "Simulation of Transient Effects in the Heavy Ion Fusion Injectors," Particle Accelerator Conference Proceedings, this volume (1993).

[2] D. Grote, A. Friedman, I. Haber, "Three Dimensional Simulation of Heavy Ion Fusion Beams: Recent Improvements to and Applications of WARP," Particle Accelerator Conference Proceedings, this volume (1993).

[3] S. Yu, et al, "Heavy Ion Fusion Injector Program," Particle Accelerator Conference Proceedings, this volume (1993).

[4] Bruce Langdon, LLNL, private communication (1992).

[5] Lou Reginato, LBL, private communication (1992).

[6] G. J. Caporaso and J.J. Barnard, "Beam Loading Effects in Heavy Ion Injectors," Particle Accelerator Conference Proceedings, this volume (1993). 